http://jmscr.igmpublication.org/home/ ISSN (e)-2347-176x ISSN (p) 2455-0450

crossref DOI: https://dx.doi.org/10.18535/jmscr/v9i2.46

\title{
Case report on Non Invasive Follicular thyroid neoplasm with papillary like Nuclear Feature (NIFTP), A Diagnostic Dilemma
}

\author{
Authors \\ Dr Akshita Mehta ${ }^{1}$, Dr Neha Mohindroo ${ }^{2}$, Dr Shobh Mohindroo ${ }^{3}$ \\ ${ }^{1}$ JR Pathology, IGMC Shimla, ${ }^{2}$ SR KNH Shimla \\ ${ }^{3}$ Associate Professor, Pathology, IGMC Shimla \\ *Corresponding Author \\ Dr Neha Mohindroo \\ SR KNH Shimla, India
}

\begin{abstract}
Non-invasive Follicular thyroid neoplasm with papillary like nuclear features (NIFTP) is a newer addition in WHO 2017 blue book of endocrine neoplasm and has an indolent behavior. We present a case of a 30 years old female who presented with neck swelling slightly left to the midline. On USG a solitary nodule was identified in left lobe of thyroid with TIRADS5. On FNA a cytological diagnosis of papillary neoplasm was given and she underwent left lobectomy post which she was given histopathological diagnosis of NIFTP. It is a difficult entity to diagnose on FNA / biopsy until the entire nodule is submitted along with the capsule so overdiagnosis and over treatment is a risk.
\end{abstract}

\section{Introduction}

Non-invasive Follicular thyroid neoplasm with papillary like nuclear features (NIFTP) is a newly added variant of follicular neoplasm with very low malignant potential. ${ }^{1}$ NIFTP presents mostly by detection of a nodule on routine examination or incidentally on imaging. Histopathology shows solid, well-circumscribed or encapsulated nodule. Diagnosis is made by strict inclusion and exclusion criteria following submission of the entire nodule with capsule for histology It is a diagnostic dilemma to report due to high incidence of over diagnosis on FNA and even on biopsy as papillary or follicular carcinoma. But diagnosis of NIFTP should be made only on the surgical specimen after careful evaluation of capsular/ lymphovascular invasion.

\section{Case Report}

A 30 years old woman presented with swelling in midline of neck for the last 2 years. On examination of the neck a single swelling measuring $(4 \times 3) \mathrm{cms}$ in midline of neck slightly towards left was observed, which was firm, non tender and moved with deglutition. TFT:T3 was raised and TSH reduced. On USG of thyroid, left lobe showed a well defined hetrogenousiso to hyperechoic solid cystic nodule with raised vascularity $(3 \times 3.5 \times 4.5) \quad \mathrm{cm}$. Radiological diagnosis of left thyroid lobe nodule, TIRADS 5 was given. Following USG, FNA was performed from a private lab and diagnosis of papillary neoplasm of thyroid was given post which she underwent left thyroidectomy and we received the specimen for histopathological examination. 
Grossly, left lobe of thyroid measured $(5 \times 4.5 \times 2.5)$ $\mathrm{cm}$. On cut section there was a well circumscribed nodule $(4.5 \times 4 \times 2.5) \mathrm{cm}$, gray white to gray brown with focal tan brown areas. Microscopically, left lobe showed encapsulated neoplasm containing closely packed variable sized follicles and focal solid sheets of cuboidal to low columnar cells having round to elongated nucleus with some cells showing nuclear enlargement, crowding, irregular nuclear contours, chromatin clearing, nuclear grooving, pseudoinclusions noted with nuclear score 2. Follicles were filled with dark colloid and follicles cleft from stroma were noted. However, no psammomma bodies, tumor necrosis and mitosis were absent. Regrossing was done, entire nodule and capsule was submitted. Papillary architecture $(<1 \%)$ and solid areas $(<30 \%)$ with no capsular/ lymphovascular invasion seen. Hence, the final diagnosis of Non-invasive Follicular thyroid neoplasm with papillary like nuclear features (NIFTP) was given.

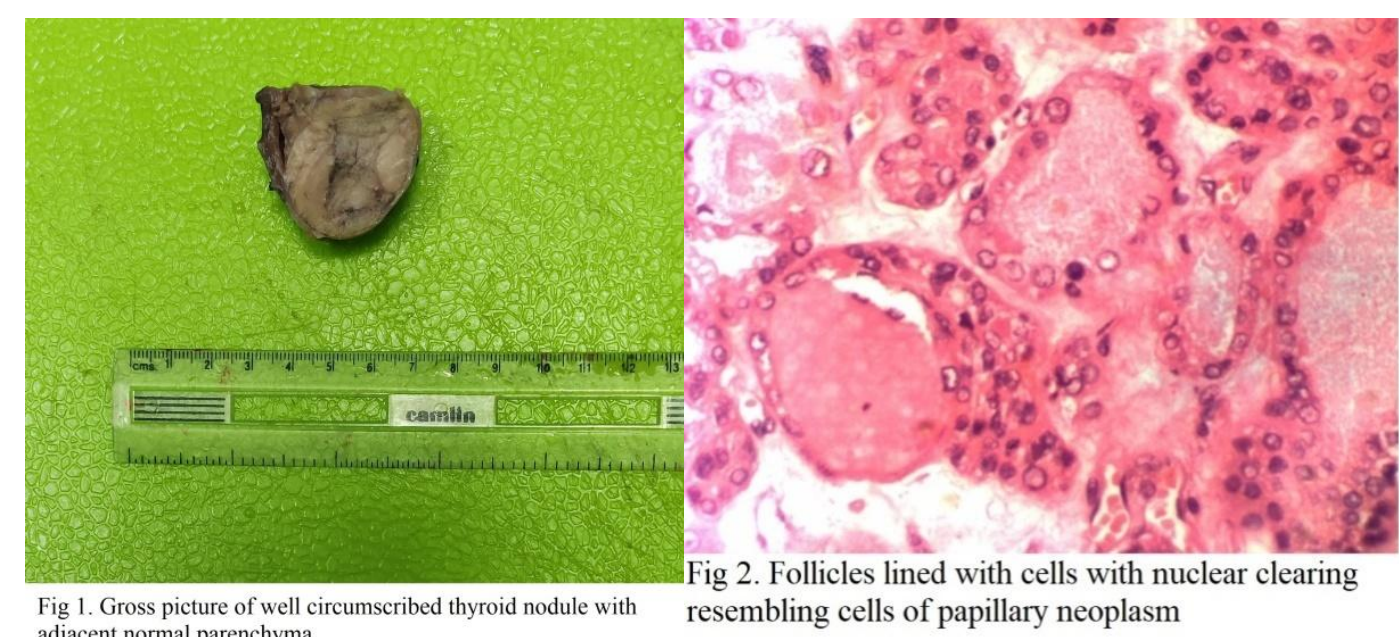

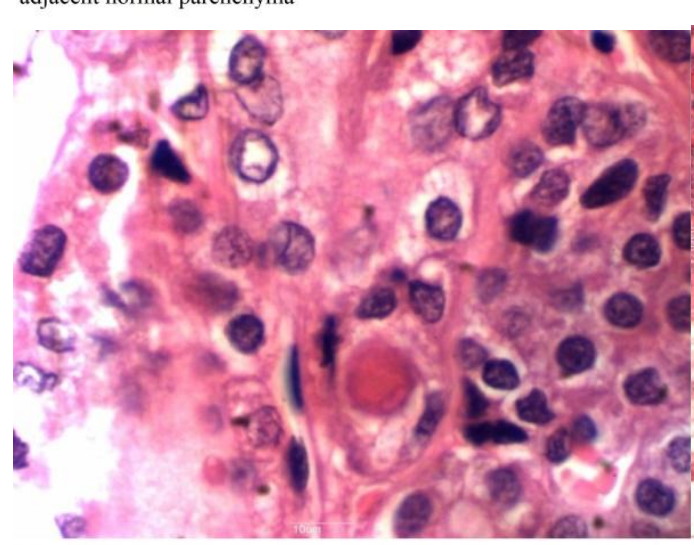

Fig. 3 Cells showing chromatin clearing and nuclear pseudoinclusion
Fig. 4 revealing capsule and outside showing cells of parathyroid gland.

\section{Discussion}

NIFTP is a non-invasive neoplasm of thyroid follicular cells with a follicular growth pattern and nuclear features of papillary thyroid carcinoma (PTC) that has an extremely low malignant potential. ${ }^{1}$ Formerly known as "noninvasive encapsulated follicular variant of papillary thyroid carcinoma (EFVPTC) ${ }^{2}$ and was introduced as NIFTP in 2017 WHO classification of thyroid tumor. ${ }^{3}$ The main goal of the introduction of
NIFTP category was to prevent over diagnosis and overtreatment.

These tumors do not show BRAF V600E mutations like papillary tumors but associated with RAS mutation as seen in Follicular tumors of thyroid. On USG, typically exhibit a circumscribed oval or round nodule with a hypoechoic rim and a hypervascular Doppler signal. ${ }^{5}$ Multifocality and bilaterality are part of the spectrum of NIFTPs, that can arise in a 
multinodular background with variable sizes from microscopic lesions to very large ones. ${ }^{6}$ FNAs of NIFTP are characterized by a microfollicular architecture along with variably nuclear features of PTC, including enlargement, crowding, contour irregularities with grooves, and chromatin clearing. $^{7,8}$
Diagnosis of NIFTP is only possible in surgical specimen for evaluation of capsular invasion and requires submission of the entire nodule. Histopathological criteria for diagnosis ${ }^{1,2}$

\begin{tabular}{|c|c|}
\hline Inclusion criteria & Exclusion criteria \\
\hline 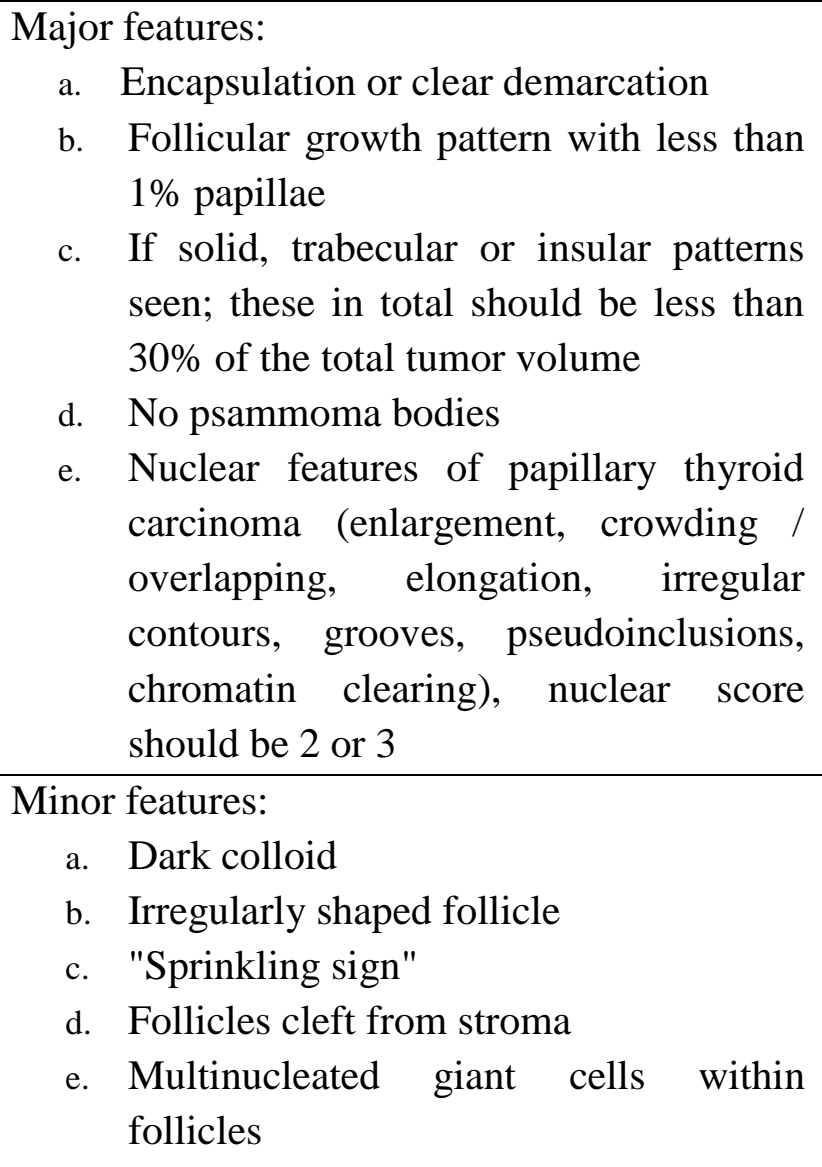 & $\begin{array}{l}\text { Any capsular or vascular invasion but if } \\
\text { the whole capsule has not been examined } \\
\text { thoroughly then the default diagnosis is } \\
\text { still noninvasive encapsulated FVPTC } \\
\text { (EFVPTC) and it is NOT a NIFTP } \\
\text { True papillary structures in more than 1\% } \\
\text { of tumor volume, psammoma bodies, } \\
\text { infiltrative border } \\
\text { Tumor necrosis (not associated with } \\
\text { FNA), increased mitoses (defined as at } \\
\text { least } 3 \text { per } 10 \text { HPF) } \\
\text { Cell / morphological characteristics of any } \\
\text { other papillary thyroid carcinoma variant } \\
\text { (e.g., tall cell, columnar cell, cribriform } \\
\text { morular, diffuse sclerosing, etc.) or } \\
\text { oncocytic lesion }\end{array}$ \\
\hline
\end{tabular}

Surgery is the main stay of treatment as NIFTP has nonmalignant behavior. However, identification of an associated microscopic PTC should be carefully evaluated because it could be a factor for LN metastasis or recurrence. ${ }^{9}$

\section{Conclusion}

NIFTP is a distinct entity from follicular or papillary carcinoma with features of both. However, it lacks capsular and lymphovascular invasion and has a very low malignant potential. The challenge is it's over diagnosis as papillary/ follicular carcinoma via FNA as the entire nodule must be submitted along with capsule for a diagnosis of NIFTP. And treatment is different from follicular or Papillary Carcinoma as surgery suffices the treatment of NIFTP and it does not require further surgery or CRT.

\section{Reference}

1. Lloyd RV, Osamura RY, Klöppel G, Rosai $\mathrm{J}$ (editors) WHO Classification of Tumours of Endocrine Organs, 4th edn. Lyon, France: IARC, 2017.

2. Nikiforov YE, Seethala RR, Tallini $G$ et al. Nomenclature revision for encapsulated 
follicular variant of papillary thyroid carcinoma. A paradigm shift to reduce overtreatment of indolent tumors. JAMA Oncol 2016; 2: 1023-9.

3. Kakudo, K., Bychkov, A., Bai, Y., Li, Y., Liu, Z. and Jung, C.K. (2018), The new 4th edition World Health Organization classification for thyroid tumors, Asian perspectives. Pathol Int, 68: 641-664.

4. Rivera M., Ricarte-Filho J., Knauf J., et al. Molecular genotyping of papillary thyroid carcinoma follicular variant according to its histological subtypes (encapsulated Vs infiltrative) reveals distinct BRAF and RAS mutation patterns. Modern Pathology.2010: 23 (9): 1191-1200.

5. Pusztaszeri M, Bongiovanni $M$. The impact of non-invasive follicular thyroid neoplasm with papillary-like nuclear features (NIFTP) on the diagnosis of thyroid nodules. Gland Surg. 2019 Aug;8 (Suppl 2):S86-S97. doi: 10.21037/gs.2018.12.01. PMID: 31475095; PMCID: PMC6694025.

6. Canini V, Leni D, Pincelli AI, et al. Clinical-pathological issues in thyroid pathology: study on the routine application of NIFTP diagnostic criteria. Sci Rep. 2019;9(1):13179.

7. Brandler TC Zhou F, Liu CZ, et al. Can noninvasive follicular thyroid neoplasm with papillary-like nuclear features be distinguished from classic papillary thyroid carcinoma and follicular adenomas by fine-needle aspiration? Cancer Cytopathol 2017;125:378-88.

8. Strickland KC, Vivero M, Jo VY, et al. Preoperative cytologic diagnosis of noninvasive follicular thyroid neoplasm with papillary-like nuclear features: a prospective analysis. Thyroid 2016;26: 1466-71.

9. Chereau N, Greilsamer T, Mirallié E, et al. NIFT-P: Are they indolent tumors? Results of a multi-institutional study. Surgery 2019;165:12-6. 